\title{
HER1-4 expression status correlates with the efficacy of gefitinib treatment and tumor cell proliferative activity in non-small cell lung cancer
}

\author{
MINAKO FUJITA $^{1}$, MITSUAKI ISHIDA ${ }^{2}$, NORIAKI TEZUKA ${ }^{1}$, SHOZO FUJINO $^{3}$, \\ TOHRU ASAI ${ }^{1}$ and HIDETOSHI OKABE ${ }^{2}$
}

\begin{abstract}
Departments of ${ }^{1}$ Surgery, and ${ }^{2}$ Clinical Laboratory Medicine and Division of Diagnostic Pathology, Shiga University of Medical Science, Shiga; ${ }^{3}$ Department of Surgery, University Hospital, Mizonokuchi, Teiko University School of Medicine, Kawasaki, Japan
\end{abstract}

Received November 16, 2007; Accepted January 3, 2008

\begin{abstract}
The expression of human epidermal growth factor receptor (HER) family proteins was examined in specimens of 52 non-small cell lung cancers to evaluate their significance in tumor cell proliferation and their response to gefitinib. Epidermal growth factor receptor (EGFR; HER1) protein was expressed most frequently (43/52 cases). HER2 and HER3 were detected in 13 and 18 cases, respectively. HER 4 was detected in only one case of squamous cell carcinoma. HER3 was expressed preferentially in adenocarcinomas of the papillary type. In most HER3-positive cases with or without other HER family proteins, the Ki-67 labelling index (18.2\%) was significantly lower than in HER3-negative cases $(30.6 \%)$ $(\mathrm{P}<0.05)$. Adenocarcinomas co-expressing EGFR, HER2 and HER3 frequently showed both a good response to gefitinib (6/7 cases) and a mutation of EGFR (4/6 cases). However, cases with a favourable response to gefitinib did not always express EGFR. Gefitinib appears to suppress growth signal pathways other than EGFR-tyrosine kinase activation in non-small cell lung cancers.
\end{abstract}

\section{Introduction}

Epidermal growth factor receptor (EGFR) belongs to the family of human epidermal growth factor receptor (HER) proteins, which are encoded by a group of Erb-B genes and transduce the stimulatory signal of EGF required for lung cancer proliferation (1-3). EGFR (HER1) with a mutation in its tyrosine kinase domain amplifies the EGF signal more strongly

Correspondence to: Dr Hidetoshi Okabe, Department of Clinical Laboratory Medicine and Division of Diagnostic Pathology, Shiga University of Medical Science, Tsukinowa-cho, Seta, Otsu, Shiga 520-2192, Japan

E-mail: okabe@belle.shiga-med.ac.jp

Key words: non-small cell lung cancer, epidermal growth factor receptor, human epidermal growth factor 1-4, gefitinib, Ki-67 than wild-type EGFR, and lung carcinomas with such mutations are susceptible to anti-EGFR therapy $(1,4)$. However, the functions of HER family proteins are modulated not only by gene mutation but also by interaction with other types of HER family proteins $(2,3,5)$; for example, by the formation of heterodimers containing different types of HER family proteins $(2,3)$. Accordingly, it is useful to evaluate the effect of anti-EGFR receptor agents in individual cases of lung carcinomas in relation to the expression of HER family proteins. In this study we reported the expression status of four HER family proteins in 52 lung carcinomas in relation to EGFR gene mutation and tumor cell proliferative activity.

\section{Materials and methods}

Materials. Fifty-two formalin-fixed or paraffin-embedded non-small cell lung cancer tissues from patients treated with gefinitib (Iressa, Astra Zeneca Pharmaceuticals) were selected from the surgical pathology specimens obtained during surgery or biopsy between 1993 and 2006.

\section{Methods}

Evaluation of gefitinib treatment. The efficacy of gefitinib treatment in the 52 patients was evaluated 4 weeks after the beginning of the treatment according to the Southwest Oncology Group modification of the Union Internationale Contre le Cancer/WHO criteria, and classified into the following five categories: complete response (CR), partial response (PR), partial response in non-measurable disease (PRNM), stable disease (SD) and progressive disease (PD) (6).

Immunohistochemistry. Appropriate tissue specimens for immunohistochemical analysis were selected after the histological evaluation of all the tumor tissue slides from the individual cases. Sections $(4 \mu \mathrm{m})$ from tumor tissue blocks were mounted on poly-L-lysine precoated slides and processed for immunohistochemical analysis using the primary antibodies listed in Table I. Immunohistochemical staining was carried out with an avidin-biotin immunoperoxidase staining kit (Dako Japan Co. Ltd., Kyoto, Japan) according to the manufacturer's instructions, and 3,3'diaminobenzidine tetra- 
Table I. List of the primary antibodies.

\begin{tabular}{llll}
\hline Antigen & \multicolumn{1}{c}{ Type of antibody (clone/code) } & \multicolumn{1}{c}{ Company } & Dilution \\
\hline EGFR (HER1) & Mouse IgG1 (sNCL-EGFR-384) & Novocastra Laboratories Ltd. & $1: 200$ \\
HER2 & Polyclonal rabbit (A0485) & Dako Japan Co. Ltd. & $1: 400$ \\
HER3 & Mouse IgG1 (SPG1) & Lab Vision Co. Ltd. & Pre-diluted \\
HER4 & Mouse IgG1 (Ab77) & Oncogene Research Products Co. Ltd. & $1: 100$ \\
Ki-67 & Mouse IgG1 (NCL-L-Ki67-MMI) & Novocastra Laboratories Ltd. & $1: 50$ \\
\hline
\end{tabular}

hydrochloride (DAB) was used for visualization. Before the application of primary antibodies, in slides for the staining of EGFR, HER2 and Ki-67, antigen retrieval by autoclaving at $120^{\circ} \mathrm{C}$ for $1 \mathrm{~min}$ in $0.1 \mathrm{mM}$ citrate buffer was carried out, and slides for the staining for HER3 and HER4 were digested in $0.05 \%$ pronase for $15 \mathrm{~min}$ at $37^{\circ} \mathrm{C}$.

Positive controls for immunohistochemical staining were as follows: HER1, skin; HER2, control slides for Hercep Test ${ }^{\mathrm{TM}}$ (Dako); HER3 and HER4, pancreas; Ki-67, lymph node with active germinal centers.

Immunoreactivity to HER2 was classified into the following four categories according to the criteria of the HER2 test of breast cancer (Hercep Test): 0, no staining; 1+, light staining; $2+$, moderate staining; $3+$, strong staining (7). The intensity of HER1 staining was evaluated in a similar manner; however, cases without membranous staining were classified as $1+$ when cytoplasmic staining was observed. Immunoreactivity to HER3 and HER4 was simply regarded as (+) when immunoreactivity was found in either the cytoplasm or the cell membrane. The ratio of Ki-67-positive nuclei in each case was evaluated after counting $>1000$ tumor cell nuclei.

EGFR mutation analysis. Mutations of exons 18, 19, 20 and 21 of the EGFR gene, corresponding to the tyrosine kinase domain, were analyzed using paraffin-embedded tumor tissue from 17 cases, with significant tumor size reduction after gefitinib treatment and/or cases co-expressing three HER family proteins. The PCR-Invader assay (BML Co. Ltd., Tokyo, Japan) was used to detect mutations.

Statistical analysis. The Student's t-test was used to assess the association of the Ki-67 labelling index with the expression status of HER family proteins and/or the type of tumor histology. Fisher's exact test was used to evaluate the statistical difference between the incidence of gefitinib efficacy in different histologic types of EGFR-positive carcinomas. StatView ${ }^{\mathrm{TM}}$ version 4.5 (Abacuus Concepts, Inc.) was used for this analysis.

\section{Results}

The findings are summarized in Table II. EGFR was the most frequently-expressed molecule in all types of non-small cell cancers, detected in 43 of our 52 cases. HER 2 immunoreactivity was found in 12 adenocarcinomas and one squamous cell carcinoma; however, the strength of the immunoreactivity in these 13 cases was weak $(1+$ in the Hercep Test). HER 3 was found in 18 cases, 16 of which were adenocarcinomas. Most of the HER3-positive adenocarcinomas were of the papillary type. Co-expression of EGFR, HER2 and HER3 was found in seven cases of adenocarcinomas (Fig. 1A-D), but not in other types of carcinomas. HER4 expression was found in only one case of squamous cell carcinoma, which was positive for EGFR.

Gefitinib treatment was effective in 32 cases, and EGFRpositive adenocarcinomas, including one adenosquamous carcinoma, responded well to gefitinib more frequently (26/30 cases) than the adenocarcinomas without EGFR (2/8 cases) $(\mathrm{P}<0.05)$. On the other hand, there was no correlation between the strength of immunoreactivity to EGFR and the response to gefitinib treatment in 14 non-adenocarcinomas. A significant reduction in tumor size occurred in 17 cases. Fourteen of the 17 were pure adenocarcinomas. Analysis for an EGFR gene mutation was conducted in 14 of these 17 cases and was found in 9 cases of adenocarcinoma $(9 / 14,64.3 \%)$, while other types of carcinoma (two squamous cell carcinomas and one adenosquamous carcinoma) did not have this mutation. Adenocarcinomas with EGFR, HER2 and HER3 frequently exhibited a significant reduction in tumor size (5/7 cases) (Table III). In addition, an EGFR gene mutation was common in these cases (4/6) (Table III). However, this mutation was not found in two cases expressing the three HER-family proteins, and one of these two cases had a poor response to gefitinib treatment (Table III). Two other PR cases with an EGFR gene mutation expressed HER3 in addition to EGFR (Table III). The remaining CR cases with this mutation expressed only EGFR (Table III). An EGFR gene mutation was not found in 5 cases with tumor size reduction (Table III). Two of them were positive only for EGFR, and the remaining cases did not show immunoreactivity to EGFR. The strength of immunoreactivity to EGFR was not related to the response to gefitinib (Table III).

The average Ki-67 labelling index was $19.3 \%$ (4-60\%) for 37 adenocarcinomas and $43.5 \%$ (6-80\%) for 15 nonadenocarcinomas. The Ki-67 labelling index in cases with a good response to gefitinib $(20.8 \%)$ was lower than in cases without a response $(35.2 \%)$. However, HER2 expression did not have a significant influence on the Ki-67 labelling index in lung carcinomas, even in tumors not expressing HER3, and HER3-positive adenocarcinomas had a significantly lower Ki-67 labelling index (18.2\%) than HER3-negative adenocarcinomas $(30.6 \%)(\mathrm{P}<0.05)$ (Fig. 1F). Co-expression of HER1 and/or HER2 did not have a significant effect on the Ki-67 labelling index.

\section{Discussion}

Four HER family proteins are encoded by the Erb-B gene and form dimers to exert their functions in cellular proliferation; 
Table II. Summary of immunohistochemistry and the efficacy of gefitinib.

\begin{tabular}{|c|c|c|c|c|c|c|}
\hline \multirow[b]{2}{*}{ Histology/case no. } & \multicolumn{4}{|c|}{ Expression of HER family proteins } & \multirow[b]{2}{*}{ Ki-67 labelling index (\%) } & \multirow[b]{2}{*}{ Response to gefitinit } \\
\hline & EGFR & HER2 & HER3 & HER4 & & \\
\hline \multicolumn{7}{|l|}{ Adenocarcinomas } \\
\hline 1 & $1+$ & $1+$ & + & - & 9 & PR \\
\hline 2 & $3+$ & $1+$ & + & - & 6 & PR \\
\hline 3 & $3+$ & $1+$ & + & - & 5 & PR \\
\hline 4 & $2+$ & $1+$ & + & - & 25 & PR \\
\hline 5 & $1+$ & $1+$ & + & - & 4 & PR \\
\hline 6 & $3+$ & $1+$ & + & - & 36 & SD \\
\hline 7 & $1+$ & $1+$ & + & - & 53 & PD \\
\hline 8 & $1+$ & $1+$ & - & - & 54 & SD \\
\hline 9 & $2+$ & $1+$ & - & - & 46 & SD \\
\hline 10 & $1+$ & $1+$ & - & - & 8 & SD \\
\hline 11 & $3+$ & $1+$ & - & - & 25 & SD \\
\hline 12 & $1+$ & 0 & + & - & 5 & PR \\
\hline 13 & $2+$ & 0 & + & - & 15 & PR \\
\hline 14 & $1+$ & 0 & + & - & 20 & SD \\
\hline 15 & $1+$ & 0 & + & - & 4 & SD \\
\hline 16 & $3+$ & 0 & + & - & 32 & SD \\
\hline 17 & $1+$ & 0 & + & - & 9 & SD \\
\hline 18 & $1+$ & 0 & - & - & 18 & CR \\
\hline 19 & $3+$ & 0 & - & - & 25 & PR \\
\hline 20 & $1+$ & 0 & - & - & 5 & PR \\
\hline 21 & $1+$ & 0 & - & - & 5 & PR \\
\hline 22 & $1+$ & 0 & - & - & 7 & PR \\
\hline 23 & $2+$ & 0 & - & - & 26 & PR \\
\hline 24 & $1+$ & 0 & - & - & 6 & SD \\
\hline 25 & $1+$ & 0 & - & - & 11 & SD \\
\hline 26 & $2+$ & 0 & - & - & 18 & SD \\
\hline 27 & $1+$ & 0 & - & - & 11 & SD \\
\hline 28 & $2+$ & 0 & - & - & 12 & PD \\
\hline 29 & $1+$ & 0 & - & - & 34 & PD \\
\hline 30 & 0 & $1+$ & - & - & 20 & PD \\
\hline 31 & 0 & 0 & + & - & 6 & PR \\
\hline 32 & 0 & 0 & + & - & 32 & PD \\
\hline 33 & 0 & 0 & + & - & 4 & PD \\
\hline 34 & 0 & 0 & - & - & 9 & SD \\
\hline 35 & 0 & 0 & - & - & 30 & PD \\
\hline 36 & 0 & 0 & - & - & 20 & PD \\
\hline 37 & 0 & 0 & - & - & 60 & PD \\
\hline \multicolumn{7}{|c|}{ Squamous cell carcinomas } \\
\hline 38 & $3+$ & $1+$ & - & - & 49 & PR \\
\hline 39 & $3+$ & 0 & + & - & 29 & PD \\
\hline 40 & $1+$ & 0 & - & + & 40 & SD \\
\hline 41 & $3+$ & 0 & - & - & 61 & SD \\
\hline 42 & $1+$ & 0 & - & - & 40 & PR \\
\hline 43 & $1+$ & 0 & - & - & 16 & PD \\
\hline 44 & $3+$ & 0 & - & - & 6 & PD \\
\hline 45 & $3+$ & 0 & - & - & 68 & PD \\
\hline 46 & $3+$ & 0 & - & - & 63 & PD \\
\hline 47 & $1+$ & 0 & - & - & 20 & PD \\
\hline 48 & $1+$ & 0 & - & - & 80 & PD \\
\hline 49 & 0 & 0 & - & - & 20 & PD \\
\hline \multicolumn{7}{|c|}{ Adenosquamous carcinoma } \\
\hline 50 & $2+$ & 0 & + & - & 33 & $\mathrm{PR}$ \\
\hline \multicolumn{7}{|c|}{ Large cell carcinomas } \\
\hline 51 & $3+$ & 0 & - & - & 67 & PD \\
\hline 52 & 0 & 0 & - & - & 60 & PD \\
\hline
\end{tabular}

CR, complete response; $\mathrm{PR}$, partial response; $\mathrm{SD}$, stable disease; $\mathrm{PD}$, progressive disease. 

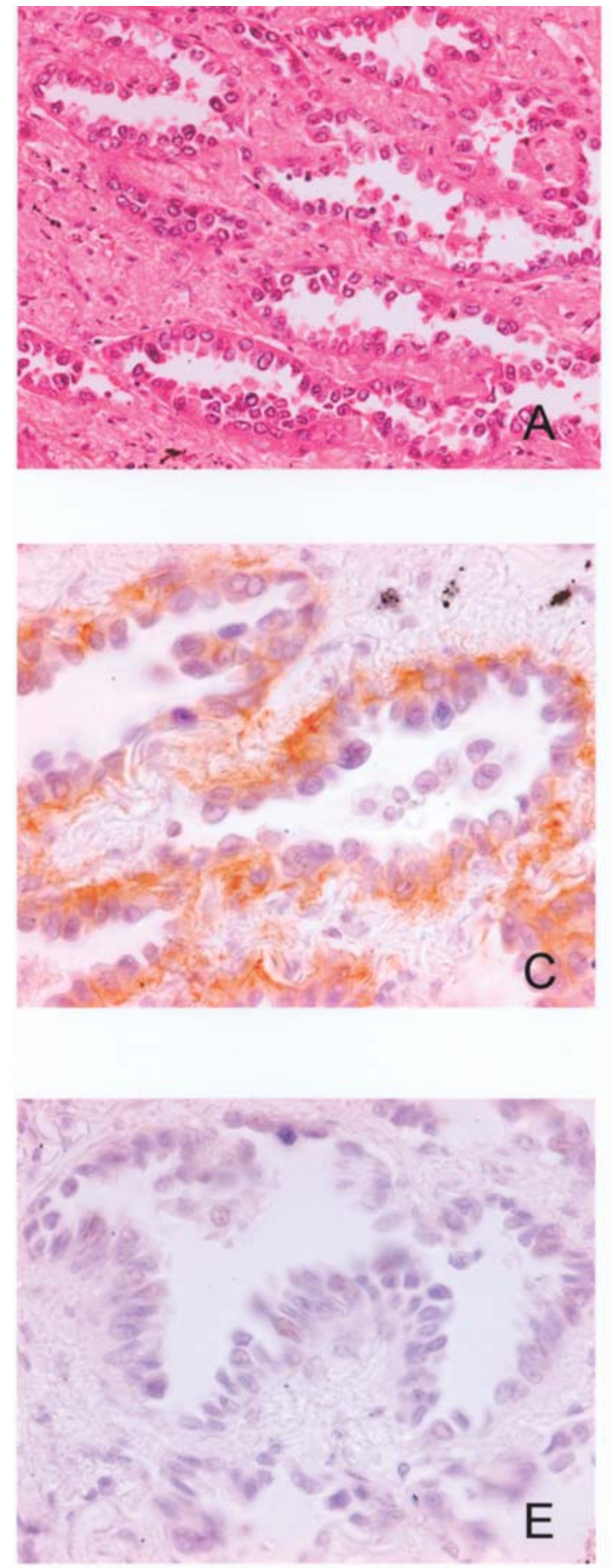
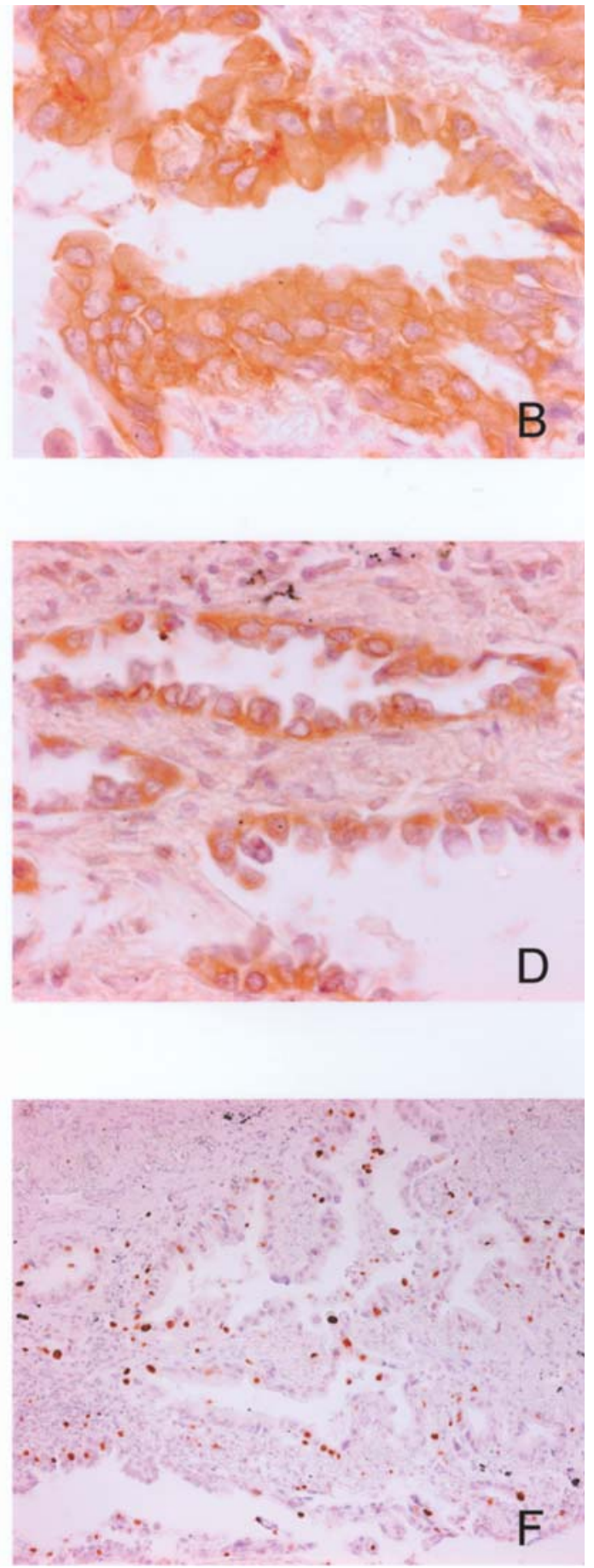

Figure 1. Papillary adenocarcinoma (case 1). (A) H\&E. (B) Diffuse strong cytoplasmic and focal discontinuous membranous staining of EGFR. (C) HER2 staining corresponding to 1+. (D) Diffuse cytoplasmic and membrane staining of HER3. (E) Absence of HER4 immunoreactivity. (F) Sporadic immunoreactivity to Ki-67 in the tumor cell nuclei. (Original magnification: A, x200; B-F, x400).

EGFR receives a signal from EGF and facilitates the proliferation of epithelial cells and their neoplasms $(2,3,5)$. In our study, EGFR was frequently detected in lung carcinomas (43/52 cases). HER 2 and/or HER3 expression was found in a considerable number of lung carcinomas, especially adenocarcinomas, though not as frequently expressed as EGFR. Most of the 18 HER3-positive adenocarcinomas had a wellorganized papillary structure, and the average Ki-67 labelling index of the HER3-positive tumors (18.2\%) was significantly lower than that of the tumors without HER3 (30.6\%) $(\mathrm{P}<0.05)$. In addition, most of the HER3-positive carcinomas (15/18 cases) co-expressed EGFR and/or HER2. These findings suggest that HER3 represses the proliferative stimuli produced by other HER family proteins (EGFR and HER2) and induces the maturation of adenocarcinoma cells.

In our analysis, 32 of 52 cases (61.5\%) showed a good response to gefitinib (CR, $\mathrm{PR}$ and $\mathrm{SD})$, with a response rate similar to that of previous reports (8). Among the EGFRpositive cases, adenocarcinomas responded more frequently $(26 / 30)$ than other types of non-small cell lung carcinomas $(5 / 14)(\mathrm{P}<0.05)$. The average Ki-67 labelling index of these 32 cases with good response $(20.8 \%)$ was significantly lower 
Table III. EGFR mutation of patients who responded to gefitinib and/or had triple expression in immunohistochemistry.

\begin{tabular}{|c|c|c|c|c|c|c|c|}
\hline \multirow[b]{2}{*}{ Case no. } & \multirow{2}{*}{$\begin{array}{l}\text { Response } \\
\text { to gefitinib }\end{array}$} & \multicolumn{4}{|c|}{ Expression of HER family proteins } & \multirow{2}{*}{$\begin{array}{l}\text { Ki-67 labelling } \\
\text { index }(\%)\end{array}$} & \multirow[b]{2}{*}{ EGFR mutation } \\
\hline & & EGFR & HER2 & HER3 & HER4 & & \\
\hline 1 & PR & $1+$ & $1+$ & + & - & 9 & Exon 21L858R \\
\hline 2 & PR & $3+$ & $1+$ & + & - & 6 & Material insufficient \\
\hline 3 & PR & $3+$ & $1+$ & + & - & 5 & Exon 21L858R \\
\hline 4 & PR & $2+$ & $1+$ & + & - & 25 & Exon 19 E746-A750 del type 1 \\
\hline 5 & PR & $1+$ & $1+$ & + & - & 4 & Exon 19 E746-A750 del type 2 \\
\hline 6 & $\mathrm{SD}$ & $3+$ & $1+$ & + & - & 36 & Not detected \\
\hline 7 & $\mathrm{PD}$ & $1+$ & $1+$ & + & - & 53 & Not detected \\
\hline 38 & PR & $3+$ & $1+$ & - & - & 49 & Not detected \\
\hline 12 & PR & $1+$ & 0 & + & - & 5 & Exon 19 E746-A750 del type 1 \\
\hline 13 & PR & $2+$ & 0 & + & - & 15 & Exon $21 \mathrm{~L} 858 \mathrm{R}$ \\
\hline 50 & PR & $2+$ & 0 & + & - & 33 & Not detected \\
\hline 18 & $\mathrm{CR}$ & $1+$ & 0 & - & - & 18 & Exon 19 E746-A750 del type 2 \\
\hline 20 & PR & $1+$ & 0 & - & - & 5 & Material insufficient \\
\hline 21 & PR & $1+$ & 0 & - & - & 5 & Not detected \\
\hline 22 & PR & $1+$ & 0 & - & - & 7 & Exon 19 L646-A750 del ins $S$ \\
\hline 23 & PR & $2+$ & 0 & - & - & 26 & Material insufficient \\
\hline 42 & PR & $1+$ & 0 & - & - & 40 & Material insufficient \\
\hline
\end{tabular}

CR, complete response; $\mathrm{PR}$, partial response; $\mathrm{SD}$, stable disease; $\mathrm{PD}$, progressive disease.

than that of cases without a good response $(35.2 \%)(\mathrm{P}<0.05)$. Seventeen of these 32 cases exhibited a significant reduction in tumor size with gefitinib treatment, and an EGFR mutation was common in cases that responded to this treatment $(9 / 11)$, as reported previously $(1,4)$. We found that cases with an EGFR mutation did not always show strong immunoreactivity to EGFR; however, cases co-expressing EGFR, HER2 and HER3 frequently exhibited significant tumor size reduction with gefitinib treatment (5/7) and frequently showed the EGFR gene mutation (4/6 cases), as also reported by Nishio et al (9). According to Scaltriti and Baselga, HER3 is required for the EGF-mediated activation of the phosphoinositide-3-kinase/ Akt pathway (10) and plays an important role in preventing apoptosis in gefitinib-sensitive carcinoma cell lines harbouring either wild-type or mutant EGFRs (11). In addition, coexpression of EGFR, HER2 and HER3 at a low-to-moderate level has been shown to promote the formation of HER2-3 heterodimers, enabling the cell to match the phenotype of cells expressing EGFR and high levels of HER2 (12). Overexpression of HER3 in EGFR-positive tumor cells in vitro induces a good response to gefitinib through the suppression of Akt activity (13), and a higher copy number of the HER2 gene was shown to correlate with a better response to gefitinib via EGFR pathways in non-small cell lung cancer patients (14). These previous reports (10-14) seem to provide a theoretical basis for the good response to gefitinib and the co-expression of HER2 and HER3 in EGFR-positive tumors shown by our analysis. However, one EGFR-positive case expressing HER2 and HER3 did not respond to gefitinib treatment, and we surmise that in this case there was another signal pathway underlying tumor proliferation that was insensitive to gefitinib.

The efficacy of gefitinib does not always seem to be dependent on the inhibition of the EGFR pathway, and one of our CR cases showed no EGFR immunoreactivity. In addition, HER3, a key factor in the gefitinib sensitivity of carcinomas expressing EGFR (11), was not demonstrated in seven EGFRpositive cases with significant tumor size reduction. These findings suggest that sensitivity to gefitinib in these eight cases did not depend on the suppression of the EGFR signal for the activation of the PI3kinase/Akt pathway mediated by HER3 (10,11). It has been demonstrated that gefitinib has an inhibitory effect on the tyrosine kinase activity of growth factor receptors other than EGFR, such as PDGFR and c-kit (15), and such effects might be responsible for the growth suppression that occurs in lung carcinomas not expressing EGFR or HER3. Further analysis of other growth factor receptors is needed to comprehensively elucidate the molecular pathways involved in the inhibitory effect of gefitinib on non-small cell lung cancers.

\section{References}

1. Lynch TJ, Bell DW, Sordella R, et al: Activating mutations in the epidermal growth factor receptor underlying responsiveness of non-small-cell lung cancer to gefitinib. N Engl J Med 350: 2129-2139, 2004.

2. Yarden $Y$ and Sliwkowski MX: Untangling the ErbB signalling network. Nat Rev Mol Cell Biol 2: 127-137, 2001.

3. Bublil EM and Yarden Y: The EGF receptor family: spearheading a merger of signaling and therapeutics. Curr Opin Cell Biol 19: 124-134, 2007.

4. Kosaka T, Yatabe Y, Endoh H, Kuwano H, Takahashi T and Mitsudomi T: Mutations of the epidermal growth factor receptor gene in lung cancer: biological and clinical implications. Cancer Res 64: 8919-8923, 2004.

5. Mendelsohn J and Baselga J: Status of epidermal growth factor receptor antagonists in the biology and treatment of cancer. J Clin Oncol 21: 2787-2799, 2003.

6. Green S and Weiss GR: Southwest Oncology Group standard response criteria, endpoint definitions and toxicity criteria. Invest New Drugs 10: 239-253, 1992. 
7. Birner P, Oberhuber G, Stani J, et al: Evaluation of the United States Food and Drug Administration-approved scoring and test system of HER-2 protein expression in breast cancer. Clin Cancer Res 7: 1669-1675, 2001.

8. Fukuoka M, Yano S, Giaccone G, et al: Multi-institutional randomized phase II trial of gefitinib for previously treated patients with advanced non-small-cell lung cancer (The IDEAL 1 Trial) [corrected]. J Clin Oncol 21: 2237-2246, 2003.

9. Nishio M, Taguchi F, Ohyanagi F, et al: Gefitinib efficacy associated with multiple expression of HER family in non-small cell lung cancer. Anticancer Res 26: 3761-3765, 2006.

10. Scaltriti $\mathrm{M}$ and Baselga J: The epidermal growth factor receptor pathway: a model for targeted Otherapy. Clin Cancer Res 12: 5268-5272, 2006.

11. Engelman JA, Jänne PA, Mermal C, et al: ErbB-3 mediates phosphoinositide 3-kinase activity in gefitinib-sensitive nonsmall cell lung cancer cell lines. Proc Natl Acad Sci USA 102: 3788-3793, 2005.
12. Shankaran H, Wiley HS and Resat H: Modeling the effects of HER/ErbB1-3 coexpression on receptor dimerization and biological response. Biophys J 90: 3993-4009, 2006.

13. Emlet DR, Schwartz R, Brown KA, Pollice AA, Smith CA and Shackney SE: HER 2 expression as a potential marker for response to therapy targeted to the EGFR. Br J Cancer 94: 1144-1153, 2006.

14. Cappuzzo F, Varella-Garcia M, Shigematsu H, et al: Increased HER2 gene copy number is associated with response to gefitinib therapy in epidermal growth factor receptor-positive non-small-cell lung cancer patients. J Clin Oncol 23: 5007-5018, 2005.

15. Mauro MJ, O'Dwyer M, Heinrich MC and Druker BJ: STI571: a paradigm of new agents for cancer therapeutics. J Clin Oncol 20: 325-334, 2002. 